\title{
Newtonian Potential and Geodesic Completeness in Infinite Derivative Gravity
}

\author{
James Edholm ${ }^{1}$ and Aindriú Conroy ${ }^{1}$ \\ ${ }^{1}$ Consortium for Fundamental Physics, \\ Physics Avenue, Lancaster University, \\ Lancaster, LA1 4YB, United Kingdom.
}

\begin{abstract}
Recent study has shown that a non-singular oscillating potential - a feature of Infinite Derivative Gravity (IDG) theories - matches current experimental data better than the standard GR potential. In this work we show that this non-singular oscillating potential can be given by a wider class of theories which allows the defocusing of null rays, and therefore geodesic completeness. We consolidate the conditions whereby null geodesic congruences may be made past-complete, via the Raychaudhuri Equation, with the requirement of a non-singular Newtonian potential in an IDG theory. In doing so, we examine a class of Newtonian potentials characterised by an additional degree of freedom in the scalar propagator, which returns the familiar potential of General Relativity at large distances.
\end{abstract}

\section{INTRODUCTION}

In the century since Albert Einstein ushered in a new paradigm for modern physics by formulating a gravitational theory that is described by the curvature of spacetime, General Relativity (GR) has withstood numerous experimental tests [1]. formulating a gravitational theory In the following century, GR It accurately describes our universe all the way down to short distances $\left(5.6 \times 10^{-5} \mathrm{~m}\right.$ from a source [2] ${ }^{1}$ and has been found to be in agreement with experimental tests on gravitational redshift and the equivalence principle [3]. More recently, the detection of gravitational waves lends more weight to this already colossal theory [4]. However, that is not to say that GR does not have any shortcomings. At the classical level, the theory breaks down at short distances in its description of black holes, for instance. GR also cannot be made geodesically past-complete, when an appropriate energy condition is met, indicating the presence of an initial singularity in the theory [5-9].

In a geodesically-incomplete spacetime, causal geodesic congruences converge to a point in a finite 'time' (affine parameter). In such a scenario a freely falling particle or photon will simply cease to exist in a finite 'time', which suggests a serious physical malady in the theory [10]. These shortcomings allow us to consider GR to be a first approximation to a broader theory.

This notion of extending GR, via additional curvature terms in the gravitational action, forms the basis for many modified theories of gravity.

Significant examples include local theories such as $f(R)$-gravity, which replaces the curvature scalar in the Einstein-Hilbert action with an arbitrary function $f(R)$, or Stelle's 4th derivative gravity, which can be seen as a generalisation of the Gauss-Bonnet term in that it includes tensorial as well as scalar modifications to the Einstein-Hilbert action. However, these finite higherderivative models still come hand-in-hand with a number

\footnotetext{
${ }^{1}$ Dark energy notwithstanding
}

of weaknesses. Stelle's theory, while perturbatively renormalizable [11], suffers from the introduction of ghosts physical excitations, characterised by negative kinetic energy [12]. $f(R)$-gravity, in comparison, may avoid the introduction of ghosts [3] but breaks down at short distances, meaning that the theory can not be said to be UV-complete [13]. In contrast, Infinite Derivative Gravity (IDG) offers a means of 'completing' GR in the UV regime (short distances). IDG is characterised by an action containing an infinite series of d'Alembertian operators $\left(\square=g^{\mu \nu} \nabla_{\mu} \nabla_{\nu}\right)$ acting on the curvature along with the mass scale of the theory, $M$.

IDG is inspired by similar infinite derivative actions used in string theory [14]. The action was first applied to gravity in [15], where the form of the modified propagator was also calculated around a Minkowski background, see also $[16,17]$. The full non-linear equations of motion were computed in [18] and the boundary terms found in [19]. The gravitational entropy for the IDG action was investigated around an (A)dS metric in [20], while the form of the (A)dS propagator was given in [21]. Constraints were put on the mass scale $M$ of IDG, either by looking at the deflection of light around the Sun [22], or by using data on the tensor-scalar ratio and spectral tilt of the Cosmic Microwave Background [23]. It has been shown that in an inflationary framework, IDG can be thought of as an extension to Starobinsky inflation [24-27].

In $[28,29]$, it was shown using a toy model of IDG that it is possible to curtail the divergences of 1-loop diagrams and show that 2-loop diagrams are finite, while in [30] the UV finiteness of IDG theories were investigated. Further work has focused on the Newtonian potential around a flat background [15, 31, 32], formulating the Hamiltonian of the IDG action [33] and avoiding cosmological singularities both through using the ansatz $\square R=c_{1} R+c_{2}[25,34]$ and via the Raychaudhuri equation [35-37].

In this paper we investigate the Newtonian potential $\Phi(r)$, describing the gravitational field of a small static spherically symmetric test mass in a flat space background. In General Relativity, this diverges according to $\Phi(r) \sim-1 / r$, becoming singular at the origin. IDG offers a means of resolving this divergence. 
It was shown in [18] that the equations of motion for IDG can be formulated in terms of two arbitrary functions of the d'Alembertian operator $a(\square)$ and $c(\square)$, which also characterise the modification to the gravitational propagator. In the case where these functions are equal, i.e. $a(\square)=c(\square)$, no additional degrees of freedom other than the massless graviton enter the system. Previous work $[14,15,31]$ has shown that, in this case, a non-singular Newtonian potential can be derived, where the potential takes the form of an error function $\Phi \sim-\operatorname{Erf}(M r / 2) / r$, for the simplest choice of $a(\square)=e^{-\square / M^{2}}$.

If we generalise this further by taking $a(\square)$ to be an exponential of a higher order polynomial, the potential is modified by an oscillating function at these higher orders. Analysis by Perivolaropoulos [2, 38] has shown that this oscillating function provides a better fit to experimental data on the force of gravity at small distances than the standard GR theory. Although further analysis is needed, there are tantalising hints that modified gravity could provide the correct description of the strength of gravity at small distances.

However, work on the avoidance of singularities in IDG theories $[35-37,39]$ has shown that we require a departure from the simple choice of $a(\square)=c(\square)$ in order for causal geodesic congruences to be made past-complete. without requiring exotic matter. The aim of the present work is to consolidate the requirements of a non-singular theory of gravity, known as the defocusing conditions, with the aforementioned converging Newtonian potential. To this end, we examine a wider class of Newtonian potentials, characterised by the condition $a(\square) \neq c(\square)$, in tandem with the defocusing conditions derived in [36] around a Minkowski background. This would allow us to avoid singularities by permitting the defocusing of null rays in a theory with a well-defined Newtonian potential at short distances.

In Section II, we give an overview of Infinite Derivative Gravity (IDG), and show how the theory offers a means of rendering null rays geodesically-complete. In Section III, we derive the Newtonian potential for IDG, and specifically look at the case which allows defocusing. Finally in Section IV we plot the Newtonian potential and interpret the results.

\section{INFINITE DERIVATIVE GRAVITY}

As mentioned in the introduction, ghosts are physical excitations bearing negative kinetic energy. These excitations are represented by a negative residue in the gravitational propagator. When interactions in such a system take place, the vacuum decays into both positive and negative energy states. This is known as the Ostrogradsky instability [41].

Previous attempts to resolve singularities by modifying gravity, such as Stelle's 4th derivative gravity [11], resulted in the introduction of ghosts, where the Hamil- tonian of the theory was unbounded from below due to the Ostrogradsky instability [41]. By adding an infinite number of derivatives to the theory this instability may be avoided through an appropriate choice of the functions $a(\square)$ and $c(\square)$. In [33] it was shown that the Hamiltonian of IDG is indeed bounded from below.

The IDG action, which is the most general, torsion-free and parity invariant action of gravity, that is quadratic in curvature was first derived in $[15,39]$

$$
\begin{aligned}
S= & \frac{1}{2} \int d^{4} x \sqrt{-g}\left(M_{p}^{2} R+R F_{1}(\square) R\right. \\
& \left.+R^{\mu \nu} F_{2}(\square) R_{\mu \nu}+C^{\mu \nu \lambda \sigma} F_{3}(\square) C_{\mu \nu \lambda \sigma}\right),
\end{aligned}
$$

where $R$ is the Ricci curvature scalar, $R_{\mu \nu}$ is the Ricci tensor, $C_{\mu \nu \lambda \sigma}$ is the Weyl tensor and $M_{p}$ is the Planck mass. Each $F_{i}(\square)=\sum_{n=0}^{\infty} f_{i_{n}} \square^{n} / M^{2 n}$ is a function of the d'Alembertian operator $\square \equiv g^{\mu \nu} \nabla_{\mu} \nabla_{\nu} . \quad M$ is the scale of modification of our theory and the $f_{i_{n}}$ are the dimensionless coefficients of the series.

The equations of motion for IDG around a Minkowski background are given by [36]

$$
\kappa T_{\mu \nu}=a(\square) R_{\mu \nu}-\frac{1}{2} \eta_{\mu \nu} c(\square) R-\frac{1}{2} f(\square) \nabla_{\mu} \nabla_{\nu} R,
$$

where we have defined ${ }^{2}$

$$
\begin{aligned}
& a(\square)=1+M_{P}^{-2}\left(F_{2}(\square)+2 F_{3}(\square)\right) \square, \\
& c(\square)=1+M_{P}^{-2}\left(-4 F_{1}(\square)-F_{2}(\square)+\frac{2}{3} F_{3}(\square)\right) \square, \\
& f(\square)=M_{P}^{-2}\left(4 F_{1}(\square)+2 F_{2}(\square)+\frac{4}{3} F_{3}(\square)\right),
\end{aligned}
$$

which abide by the constraint $a(\square)-c(\square)=f(\square) \square$. From (2) we can derive the propagator around a flat background $[15,16]$

$$
\Pi\left(p^{2}\right)=\frac{\mathcal{P}^{(2)}}{p^{2} a\left(-p^{2}\right)}-\frac{\mathcal{P}_{s}^{(0)}}{p^{2}\left(a\left(-p^{2}\right)-3 c\left(-p^{2}\right)\right)}
$$

where $\mathcal{P}^{(2)}$ is the spin-2 projection operator, $\mathcal{P}_{s}^{(0)}$ is the scalar spin-0 projection operator and $p_{\mu}=i \partial_{\mu}$ is the momentum, so that in flat space $\square=-g^{\mu \nu} p_{\mu} p_{\nu}=-p^{2}$. The simplest choice is to set $a(\square)=c(\square)$ equal to the exponential of an entire function, which by definition does not have any roots, and therefore the propagator will not receive any additional degrees of freedom other than the massless graviton. However, it was shown in [39], see also $[17,37]$, that the scalar sector of the propagator can have at most one additional pole without ghost-like degrees of

\footnotetext{
${ }^{2}$ We denote the linearised curvatures around a Minkowski background as $R, R_{\mu \nu}, R_{\mu \nu \rho \sigma}$.
} 
freedom being introduced. This allows us to write the ghost-free condition ${ }^{3}$

$$
(a(\square)-3 c(\square)) R=2\left(\square / m^{2}-1\right) \bar{a}(\square) R,
$$

where $\bar{a}(\square)$ is the exponential of an entire function and so $a\left(-p^{2}\right)-3 c\left(-p^{2}\right)$ has a single pole in the scalar sector at $\square \rightarrow-p^{2}=m^{2}$, which produces an extra spin-0 particle of mass $m$. By expanding to first order in $\square$, we find that $m^{2} \equiv M_{P}^{2} /\left(6 f_{1_{0}}-f_{2_{0}}-M_{p}^{2} / M^{2}\right)[36]$. We know that $m^{2}>0$ so that the particle has real mass and therefore no tachyons are introduced.

If either $a(\square)=c(\square)$ or (5) holds, the propagator contains an exponential term in the denominator, which suppresses the propagator at high momentum. This exponential suppression results in an exponential enhancement of the vertex factors of the relevant Feynman diagrams $[17,28,29,39]$ meaning that interaction does not take place at this point, as in a local theory. In this sense, the action can be considered to describe an effective theory.

\section{A. Defocusing conditions}

The Raychaudhuri equation is a model-independent identity which relates the geometry of spacetime to the contribution of gravity via the curvature. It says that for a null tangent vector $k_{\mu}$, satisfying $k^{\mu} k_{\mu}=0$, the expansion parameter $\theta=\nabla_{\mu} k^{\mu}$ is described by ${ }^{4}$

$$
\frac{d \theta}{d \lambda}+\frac{1}{2} \theta^{2} \leq-R_{\mu \nu} k^{\mu} k^{\nu}
$$

where $\lambda$ is the affine parameter [10]. In order to have an expansion parameter which is positive and increasing, and therefore allow the defocusing of the null rays, we require $R_{\mu \nu} k^{\mu} k^{\nu}<0$. In GR, using the Einstein equation, $G_{\mu \nu}=\kappa T_{\mu \nu}$, we cannot fulfil this condition because the Null Energy Condition (NEC) requires that $T_{\mu \nu} k^{\mu} k^{\nu} \geq$ 0. By the Hawking-Penrose Singularity Theorem [40], this inability to defocus will always lead to a singularity. To be precise, a spacetime cannot be null geodesically complete in the past direction if $R_{\mu \nu} k^{\mu} k^{\nu}>0$ [9].

However, in IDG this is not the case and we will see that it is possible to have defocusing. By contracting the equations of motion (2) with $k^{\mu} k_{\nu}$, we can see that the contribution of gravity to the Raychaudhuri equation is

$$
R_{\mu \nu} k^{\mu} k^{\nu}=\frac{1}{a(\square)}\left[\kappa T_{\mu \nu} k^{\mu} k^{\nu}+\frac{k^{\mu} k^{\nu}}{2} f(\square) \nabla_{\mu} \nabla_{\nu} R\right]
$$

${ }^{3}(a(\square)-3 c(\square)) R$ corresponds to the trace equation, found by contracting (2) with $g^{\mu \nu}$.

${ }^{4}$ We have taken the simplest case of the Raychaudhuri equation here by making two simplifications. Firstly, we take the congruence of null rays to be orthogonal to the hypersurface, so that the twist tensors vanish. Secondly, the shear tensor gives a positive contribution to the right-hand side and so we can neglect it for our purposes. which was studied in a cosmological setting [36]. When considering a static spherically symmetric perturbation around a flat background, then the defocusing condition is

$$
R_{\mu \nu} k^{\mu} k^{\nu}=\frac{1}{a(\square)}\left[\kappa T_{\mu \nu} k^{\mu} k^{\nu}+\frac{\left(k^{r}\right)^{2}}{2} f(\square) \square R(r)\right]<0,
$$

where $\square R(r)=\frac{1}{r^{2}} \partial_{r}\left(r^{2} \partial_{r}\right) R(r)$. Note that the function $a(\square)$ acting on the curvature cannot be negative, as this would lead to the Weyl ghost. This is because $a\left(-p^{2}\right)$ is the modification to the spin-2 part of the propagator, so it must be positive to avoid negative residues [16]. Therefore if the NEC holds true, we arrive at the minimum defocusing condition

$$
\frac{a(\square)-c(\square)}{a(\square)} R(r)<0 .
$$

The first observation to make is that defocusing cannot occur in the case of $a(\square)=c(\square)$, which corresponds to requiring that there are no extra poles in the scalar propagator and therefore no extra scalar propagating modes. As above, we can allow a single extra scalar propagating mode without introducing ghosts into the system $[17,37,39]$. This corresponds to adding an extra root to $a(\square)-3 c(\square)$ which from (5) means that the relationship between $a(\square)$ and $c(\square)$ is given by [36]

$$
c(\bar{\square})=\frac{a(\bar{\square})}{3}\left[1+2\left(1-\square / m^{2}\right) \tilde{a}(\bar{\square})\right],
$$

where $\tilde{a} \equiv \frac{\bar{a}(\square)}{a(\square)}$ is an exponent of an entire function. Hence the propagator is given by [36]

$$
\Pi\left(p^{2}\right)=\frac{1}{a\left(-p^{2}\right)}\left[\frac{\mathcal{P}^{(2)}}{p^{2}}+\frac{\mathcal{P}_{S}^{(0)}}{2 p^{2}\left(1+p^{2} / m^{2}\right) \tilde{a}\left(-p^{2}\right)}\right],
$$

while the minimum condition for null rays to defocus (9) becomes

$$
\left(1-\square / m^{2}\right) \tilde{a}(\square) R(r)>R(r) .
$$

\section{NEWTONIAN POTENTIAL}

In this section, we describe the general method for finding the Newtonian Potential, which was previously studied for the more specific case when $a(\square)=c(\square)[15,31$, 42], and we will generalise that to the case when $a(\square) \neq$ $c(\square)$. When we take the metric generated by a small static spherically symmetric test mass added to a flat space background, following the method of [15, 31, 42],

$$
d s^{2}=-(1+2 \Phi(r)) d t^{2}+(1-2 \Psi(r)) \eta_{i j} d x^{i} d x^{j},
$$

this is akin to perturbing the flat space metric $\eta_{\mu \nu}$ as $g_{\mu \nu}=\eta_{\mu \nu}+h_{\mu \nu}$, where

$$
h_{00}=h^{00}=-2 \Phi \quad \text { and } \quad h_{i j}=h^{i j}=-2 \Psi \eta_{i j} .
$$


As a result, the scalar curvature and 00 component of the Ricci curvature tensor around the flat Minkowski background are given by

$$
R=4 \Delta \Psi(r)-2 \Delta \Phi(r), \quad R_{00}=\Delta \Phi(r),
$$

where $\Delta \equiv \eta^{i j} \partial_{i} \partial_{j}$ is the Laplace operator.

Then from the equations of motion (2), the trace and 00 component equations of motion are ${ }^{5}$

$$
\begin{aligned}
& -\kappa \rho=\kappa T=\frac{1}{2}(a(\square)-3 c(\square)) R, \\
& \kappa \rho=\kappa T_{00}=a(\square) R_{00}+\frac{1}{2} c(\square) R .
\end{aligned}
$$

Here we have taken the weak field approximation so that $\rho \gg p$, where $\rho$ is the density and $p$ is the pressure of the test mass. Therefore $T=(-\rho+3 p) \approx-\rho$ and $T_{00} \approx \rho$. Combining this with (15) leads to

$$
\begin{aligned}
& \Delta \Psi(r)=-\frac{c(\square)}{(a(\square)-2 c(\square))} \Delta \Phi(r), \\
& \Delta \Phi(r)=\frac{a(\square)-2 c(\square)}{a(\square)(a(\square)-3 c(\square))} \kappa \rho .
\end{aligned}
$$

As our point source is of mass $\mu$, its density is approximated by a 3-dimensional Dirac-delta function: $\rho=$ $\mu \delta^{3}(\boldsymbol{r})$. Next we use a Fourier transform to calculate $\Phi(r)$ by using the same method as for calculating the Coulomb potential [43, 44].

When the Dirac-delta function is Fourier transformed into momentum space or $p$-space, it becomes [15]

$$
\rho=\mu \delta^{3}(\boldsymbol{r})=\mu \int \frac{d^{3} p}{(2 \pi)^{3}} e^{i \boldsymbol{p} \cdot \boldsymbol{r}}
$$

As we go into momentum space, we take $\square \rightarrow-p^{2}$, giving

$$
\begin{aligned}
\Phi(r) & =-\frac{\kappa \mu}{(2 \pi)^{3}} \int_{-\infty}^{\infty} d^{3} p \frac{a-2 c}{a(a-3 c)} \frac{e^{i \boldsymbol{p} \cdot \boldsymbol{r}}}{p^{2}} \\
& =-\frac{\kappa \mu}{4 \pi^{2} r} \int_{-\infty}^{\infty} d k \frac{(a-2 c)}{a(a-3 c)} \frac{\sin (p r)}{p} .
\end{aligned}
$$

Trivially, we can use (17) to see that

$$
\Psi(r)=\frac{\kappa \mu}{4 \pi^{2} r} \int_{-\infty}^{\infty} d p \frac{c}{a(a-3 c)} \frac{\sin (p r)}{p} .
$$

Note that if we set $a\left(-p^{2}\right)=c\left(-p^{2}\right)$,

$$
\Phi(r)=\Psi(r)=-\frac{\kappa \mu}{4 \pi^{2} r} \int_{-\infty}^{\infty} d p \frac{1}{2 a} \frac{\sin (p r)}{p} .
$$

This case was discussed in [15, 31]. For the simplest case, where $a(\square)=e^{-\square / M^{2}}$, it results in the $1 / r$ fall of the potential seen in GR being modified by the error function,

\footnotetext{
5 We have noted that the time derivatives of $R(r)$ and $R_{00}(r)$ vanish.
}

i.e. $\Phi(r) \sim \frac{\operatorname{Erf}(r)}{r}$. For large $r, \operatorname{Erf}(r) \approx 1$ but at short distances, $\operatorname{Erf}(r) \sim r$, so $\Phi(r) \sim 1$. Therefore the $1 / r$ behaviour is retained at large distances but at short distances, the modification means that the potential simply tails off to a constant and the potential is no longer singular.

\section{A. Potential for IDG with defocusing}

In this paper we will extend the calculation of the IDG potential to the case where $a \neq c$, which allows us to avoid singularities. We describe the relationship between $a(\square)$ and $c(\square)$ using (10), and by inserting (10) into (19) we find that

$$
\begin{aligned}
& \Phi(r)=-\frac{\kappa \mu}{24 \pi^{2} r} \\
& \quad \times \quad \int_{-\infty}^{\infty} d p\left[4-\frac{m^{2}}{\tilde{a}\left(-p^{2}\right)\left(m^{2}+p^{2}\right)}\right] \frac{\sin (p r)}{p a\left(-p^{2}\right)} .
\end{aligned}
$$

The calculation we need to perform is

$$
f(r)=\int_{-\infty}^{\infty} d p\left[4-\frac{m^{2}}{\tilde{a}\left(-p^{2}\right)\left(m^{2}+p^{2}\right)}\right] \frac{\sin (p r)}{p a\left(-p^{2}\right)},
$$

where $\Phi(r) \sim-\frac{f(r)}{r}$. We can write $\tilde{a}\left(-p^{2}\right)=e^{\tau\left(-p^{2}\right)}$ and $a\left(-p^{2}\right)=e^{\gamma\left(-p^{2}\right)}$, and this results in

$$
f(r)=\int_{-\infty}^{\infty} d p\left[4-\frac{m^{2} e^{-\tau\left(-p^{2}\right)}}{m^{2}+p^{2}}\right] \frac{e^{-\gamma\left(-p^{2}\right)} \sin (p r)}{p}
$$

This is our main result - we have shown that we can have defocusing as well as a non-singular Newtonian potential. This potential returns to the GR value in the infrared limit, i.e for large values of $r .^{6}$

\section{B. Conditions on $a\left(-p^{2}\right)$ and $\tilde{a}\left(-p^{2}\right)$}

Next we investigate the conditions that must be placed on $a\left(-p^{2}\right)$ and $\tilde{a}\left(-p^{2}\right)$, and therefore what we can say about $\tau\left(-p^{2}\right)$ and $\gamma\left(-p^{2}\right)$.

First we look at the spin-0 part of the propagator. From (11), we have

$$
\Pi\left(p^{2}\right)=\frac{1}{e^{\gamma\left(-p^{2}\right)}}\left[\frac{P^{(2)}}{p^{2}}+\frac{P^{(0)}}{2 p^{2}\left(1+p^{2} / m^{2}\right) e^{\tau\left(-p^{2}\right)}}\right],
$$

where $P^{(2)}$ is the spin-2 projection operator and $P^{(0)}$ is the spin-0 projection operator. We require that in the UV, the propagator is exponentially suppressed, allowing us the possibility of curtailing divergences [28]. From the spin-0 sector of (25), we observe the condition $\gamma\left(-p^{2}\right)+$

\footnotetext{
6 Note that in the limit $M \rightarrow \infty$ we return to a non-local theory, which is fourth-order gravity [45].
} 
$\tau\left(-p^{2}\right)>0$. Fortunately, this condition ensures that the integral (24) converges. Note that a priori, it is possible that there are coefficients in front of the exponentials $e^{\gamma\left(-p^{2}\right)}$ and $e^{\tau\left(-p^{2}\right)}$, but by requiring that we return to the GR propagator in the infrared limit $k \ll m, M$, we are obliged to set the coefficients to 1 . Also, by requiring the spin-2 part of the propagator (25) to be exponentially suppressed we have $\gamma\left(-p^{2}\right)>0$.

From looking at (12) in momentum space, the defocusing condition imposes a constraint: $\tau\left(-p^{2}\right) \geq 0$. Thus, we have the functions $a\left(-p^{2}\right)=e^{\gamma\left(-p^{2}\right)}$ and $\tilde{a}\left(-p^{2}\right)=$ $e^{\tau\left(-p^{2}\right)}$ where $\gamma\left(-p^{2}\right)$ is positive function and $\tau\left(-p^{2}\right)$ is a non-negative function. In fact, the result of these constraints is that the integral (24) converges.

We therefore have two free mass scales $M$ and $m$ and two unspecified functions $\tau\left(-p^{2}\right)$ and $\gamma\left(-p^{2}\right)$. We will henceforth choose both the mass scales to be the Planck mass $M_{P}$ and choose the simplest possible version of $\tau\left(-p^{2}\right)$, which is $\tau=0$. This choice means that all of the freedom in the model is tied up in the function $\gamma\left(-p^{2}\right)$, which we will choose to be the monomial $\gamma\left(p^{2}\right)=\left(C p^{2} / M_{P}^{2}\right)^{D}$. We therefore have only two free parameters, $C$ and $D$.

\section{PLOTTING THE RESULTS}

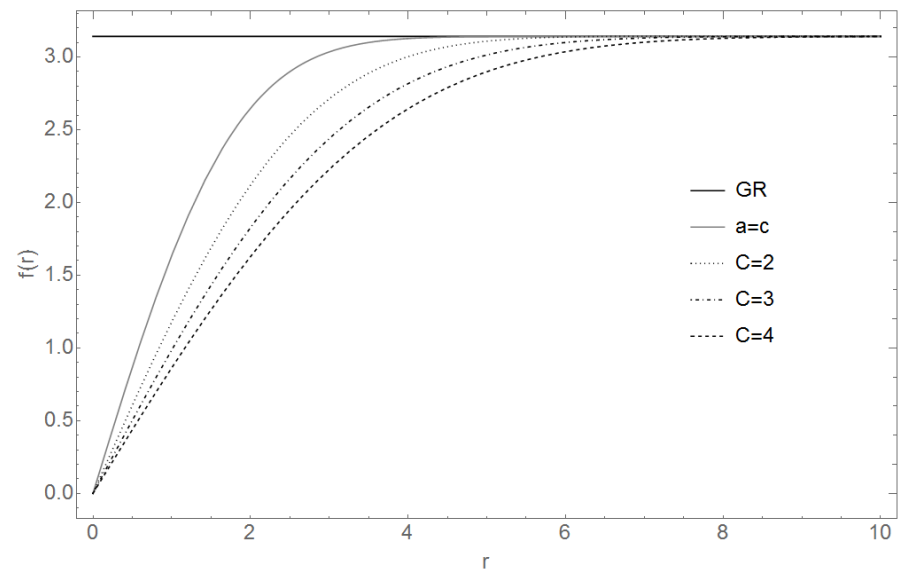

FIG. 1: We plot $f(r)$ vs $r$ for different $C$ and $D=1$, where $\gamma\left(p^{2}\right)=\left(C p^{2} / M_{P}^{2}\right)^{D}$. See (26). For illustrative purposes, we have taken $M_{p}=1 \mathrm{~m}^{-1}$. We compare our results to the $a(\square)=c(\square)$ case seen in (21). Here, we see that as $C$ increases, the effect of IDG can be seen increasingly further away from the origin.

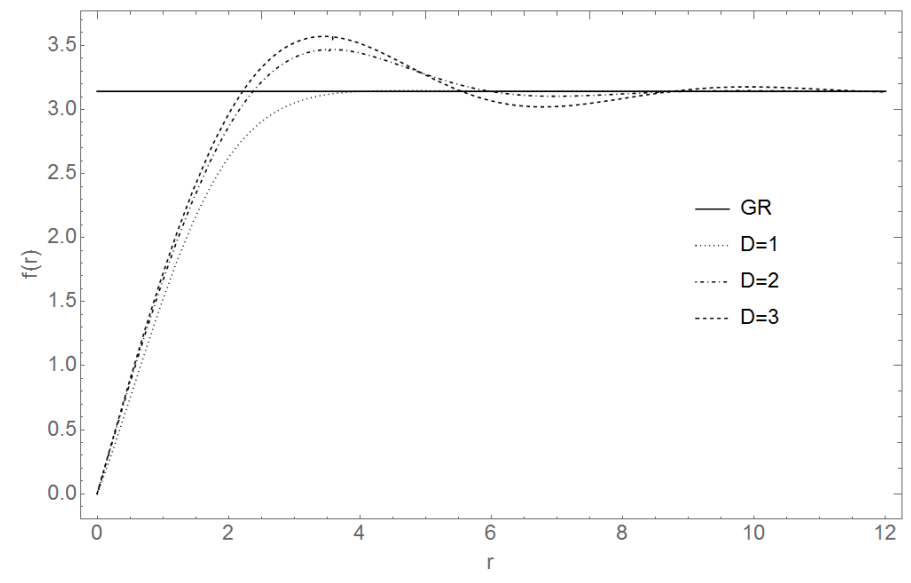

FIG. 2: We plot $f(r)$ vs $r$ for different $D$ and $C=1$ where $\gamma\left(p^{2}\right)=\left(C p^{2} / M_{P}^{2}\right)^{D}$. See (26). The plot shows that for $D>1$ the potential oscillates. As the value of

$D$ increases, so too does the magnitude of these oscillations. For illustrative purposes, we have taken $M_{p}=1 \mathrm{~m}^{-1}$ and again we find that our results reduce to that of GR at large distances.

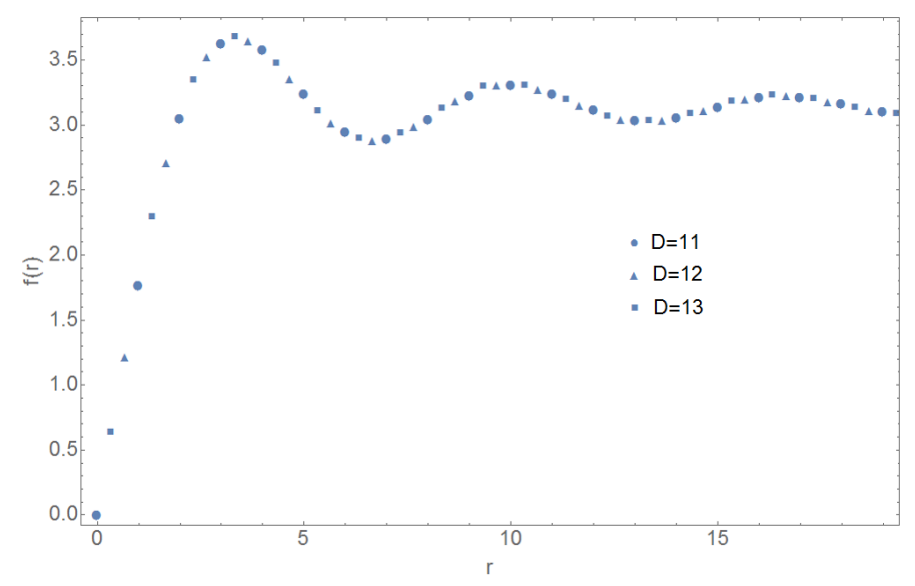

FIG. 3: We plot $f(r)$ vs $r$ for different $D$ greater than 10 with $C=1$, where $\gamma\left(p^{2}\right)=\left(C p^{2} / M_{P}^{2}\right)^{D}$. See (26).

We can see that for $D>10$, increasing $D$ does not affect the potential. For illustrative purposes, we have taken $M_{p}=1 \mathrm{~m}^{-1}$. We can parameterise these curves as $\alpha_{1} r$ for $r<1$ and $\alpha_{2} \cos \left(\theta r+\theta_{0}\right) / r$ for $r>1$, where $\alpha_{1}$, $\alpha_{2}, \theta$ and $\theta_{0}$ are constants, as in [38]. 


\section{A. Choosing a form for $a\left(-p^{2}\right)$ and $\tilde{a}\left(-p^{2}\right)$}

For the simplest choice $\tau\left(-p^{2}\right)=0$ and $\gamma\left(p^{2}\right)=$ $\left(C p^{2} / M_{P}^{2}\right)^{D}$, this gives ${ }^{7}$

$f(r)=\int_{-\infty}^{\infty} d p\left[4-\frac{M_{P}^{2}}{M_{P}^{2}+p^{2}}\right] \frac{e^{-\left(C p^{2} / M_{P}^{2}\right)^{D}} \sin (p r)}{p}$.

In Fig. 1 , we take $D=1$ and plot $f(r)$ for different choices of $C$ using (26). Clearly increasing the value of $C$ moves the point at which the non-locality kicks in further away from the origin. Next in Fig. 2 we look at how the potential varies for the same $C$ but with different values of $D$, which is the power of $p^{2}$ in (26).

As we increase the value of $D$, our potential begins to oscillate, as was found in [31]. These oscillations grow in size as $D$ increases until about $D=10$.

This is because

$$
\lim _{D \rightarrow \infty} e^{-\left(C p^{2} / M_{P}^{2}\right)^{D}}=\operatorname{rect}\left(C p^{2} / M_{P}^{2}\right),
$$

where $\operatorname{rect}(x)$ is the rectangle function, which is defined by $\operatorname{rect}(x)=1$ for $|x|<1$ and $\operatorname{rect}(x)=0$ for $|x|>1$. For $D>10,(27)$ is a very good approximation and so increasing the value of $D$ does not change the potential.

Our next task is to investigate the choice of large powers of $p^{2}$ which has been shown to fit recent experimental data [38] at small distances for the $a(\square)=c(\square)$ case. We will see whether we can still obtain oscillating solutions with conditions necessary to realise defocusing. In Fig. 3 we plot (26) for different choices of $D>10$ and note that it can still be parameterised accurately as

$$
f(r)= \begin{cases}\alpha_{1} r & \text { for } 0<r<1, \\ 1+\alpha_{2} \frac{\cos \left(\theta r+\theta_{0}\right)}{r} & \text { for } 1<r .\end{cases}
$$

In other words, the oscillating solution which was hinted at by experimental data can be produced by a modified gravity solution which also allows geodesic completeness.

\section{CONCLUSION}

We have found the Newtonian potential for a wider class of Infinite Derivative Gravity (IDG) theories than were previously investigated and analysed various cases of the theory. Analysis of data from experimental tests has hinted that, at small distances, an oscillating nonlocal potential provides the best fit to experimental data. We have shown that an IDG theory constrained to allow the defocusing of null rays, and therefore geodesic completeness,

still produces a non-singular potential which returns to the standard GR result at large distances. This result can still be parameterised as the oscillating function which provides a good fit to the data.

By allowing defocusing, it is necessary to introduce extra parameters into the model, although we can reduce the freedom in these parameters by making appropriate choices about the mass scales and the form of the functions.

Our results can be tested experimentally, which will allow us to put constraints on our parameters. Future research could look at moving away from the minimal model and reintroducing the choice of parameters which we reduced, or finding the potential around a de Sitter background rather than a flat space background.

\section{ACKNOWLEDGEMENTS}

The authors would like to thank David Burton and Anupam Mazumdar for their invaluable help in preparing this paper.
[1] C. M. Will, Living Rev. Relativity, "The Confrontation between General Relativity and Experiment" 17, (2014), 4

[2] D. J. Kapner, T. S. Cook, E. G. Adelberger, J. H. Gundlach, B. R. Heckel, C. D. Hoyle and H. E. Swanson, "Tests of the gravitational inverse-square law below the darkenergy length scale," Phys. Rev. Lett. 98 (2007) 021101 doi:10.1103/PhysRevLett.98.021101 [hep-ph/0611184].

[3] T. Clifton, P. G. Ferreira, A. Padilla and C. Skordis, "Modified Gravity and Cosmology," Phys. Rept. 513 (2012) 1 doi:10.1016/j.physrep.2012.01.001 [arXiv:1106.2476 [astro-ph.CO]].

\footnotetext{
7 Note that for large $r$, then $\Psi(r)=\Phi(r)$, i.e. the Eddington parameter $-\Phi / \Psi$ is equal to one, as expected from experimental bounds using data from the Cassini probe [46].
}

[4] B. P. Abbott et al. [LIGO Scientific and Virgo Collaborations], "Observation of Gravitational Waves from a Binary Black Hole Merger," Phys. Rev. Lett. 116 (2016) no.6, 061102 [arXiv:1602.03837 [gr-qc]].

[5] R. P. Geroch, "What is a singularity in general relativity?," Annals Phys. 48 (1968) 526. doi:10.1016/00034916(68)90144-9

[6] S. Kar, "An introduction to the Raychaudhuri equations," Resonance J. Sci. Educ. 13 (2008) 319. doi:10.1007/s12045-008-0013-1

[7] A. Vilenkin and A. C. Wall, "Cosmological singularity theorems and black holes," Phys. Rev. D 89 (2014) no.6, 064035 doi:10.1103/PhysRevD.89.064035 [arXiv:1312.3956 [gr-qc]].

[8] T. Vachaspati and M. Trodden, Phys. Rev. D 61 (1999) 023502 doi:10.1103/PhysRevD.61.023502 [grqc/9811037]. 
[9] S. W. Hawking and G. F. R. Ellis. "The Large Scale Structure of Space-Time" Cambridge Monographs on Mathematical Physics. Cambridge University Press, (2011)

[10] R. M. Wald, "General Relativity", Chicago, Usa: Univ. Pr. (1984) 491p

[11] K. S. Stelle, Phys. Rev. D 16, 953 (1977) "Renormalization of higher-derivative quantum gravity"

[12] P. Van Nieuwenhuizen, "On ghost-free tensor lagrangians and linearized gravitation," Nucl. Phys. B 60 (1973) 478. doi:10.1016/0550-3213(73)90194-6

[13] D. Blas, "Aspects of Infrared Modifications of Gravity," arXiv:0809.3744 [hep-th].

[14] A. A. Tseytlin, "On singularities of spherically symmetric backgrounds in string theory," Phys. Lett. B 363 (1995) 223 doi:10.1016/0370-2693(95)01228-7 [hep-th/9509050].

[15] T. Biswas, E. Gerwick, T. Koivisto and A. Mazumdar, "Towards singularity and ghost free theories of gravity," Phys. Rev. Lett. 108, 031101 (2012) doi:10.1103/PhysRevLett.108.031101 [arXiv:1110.5249 [gr-qc]].

[16] T. Biswas, T. Koivisto and A. Mazumdar, "Nonlocal theories of gravity: the flat space propagator," arXiv:1302.0532 [gr-qc].

[17] L. Buoninfante, "Ghost and singularity free theories of gravity," arXiv:1610.08744 [gr-qc].

[18] T. Biswas, A. Conroy, A. S. Koshelev and A. Mazumdar, "Generalized ghost-free quadratic curvature gravity," Class. Quant. Grav. 31 (2014) 015022 Erratum: [Class. Quant. Grav. 31 (2014) 159501] doi:10.1088/02649381/31/1/015022, 10.1088/0264-9381/31/15/159501 [arXiv:1308.2319 [hep-th]].

[19] A. Teimouri, S. Talaganis, J. Edholm and A. Mazumdar, "Generalised Boundary Terms for Higher Derivative Theories of Gravity," JHEP $\mathbf{1 6 0 8}$ (2016) 144 doi:10.1007/JHEP08(2016)144 [arXiv:1606.01911 [grqc]l.

[20] A. Conroy, A. Mazumdar, S. Talaganis and A. Teimouri, "Nonlocal gravity in D dimensions: Propagators, entropy, and a bouncing cosmology," Phys. Rev. D 92 (2015) no.12, 124051 doi:10.1103/PhysRevD.92.124051 [arXiv:1509.01247 [hep-th]].

[21] T. Biswas, A. S. Koshelev and A. Mazumdar, "Consistent higher derivative gravitational theories with stable de Sitter and anti de-Sitter backgrounds," Phys. Rev. D 95 (2017) no.4, 043533 doi:10.1103/PhysRevD.95.043533 [arXiv:1606.01250 [gr-qc]].

[22] L. Feng, "Light Bending in the Infinite Derivative Theories of Gravity," arXiv:1703.06535 [gr-qc].

[23] J. Edholm, "UV completion of the Starobinsky model, tensor-to-scalar ratio, and constraints on nonlocality," Phys. Rev. D 95 (2017) no.4, 044004 doi:10.1103/PhysRevD.95.044004 [arXiv:1611.05062 [gr$\mathrm{qc}]]$.

[24] F. Briscese, A. Marcian, L. Modesto and E. N. Saridakis, "Inflation in (Super-)renormalizable Gravity," Phys. Rev. D $\mathbf{8 7}$ (2013) no.8, 083507 doi:10.1103/PhysRevD.87.083507 [arXiv:1212.3611 [hep-th]].

[25] T. Biswas, A. S. Koshelev, A. Mazumdar and S. Y. Vernov, "Stable bounce and inflation in non-local higher derivative cosmology," JCAP 1208 (2012) 024 doi:10.1088/1475-7516/2012/08/024 [arXiv:1206.6374 [astro-ph.CO]].
[26] B. Craps, T. De Jonckheere and A. S. Koshelev, JCAP 1411 (2014) no.11, 022 doi:10.1088/14757516/2014/11/022 [arXiv:1407.4982 [hep-th]].

[27] A. S. Koshelev, L. Modesto, L. Rachwal and A. A. Starobinsky, "Occurrence of exact $R^{2}$ inflation in non-local UV-complete gravity," JHEP 1611 (2016) 067 doi:10.1007/JHEP11(2016)067 [arXiv:1604.03127 [hepth]].

[28] S. Talaganis, T. Biswas and A. Mazumdar, "Towards understanding the ultraviolet behavior of quantum loops in infinite-derivative theories of gravity," Class. Quant. Grav. 32 (2015) no.21, 215017 doi:10.1088/02649381/32/21/215017 [arXiv:1412.3467 [hep-th]].

[29] S. Talaganis, "Quantum Loops in Non-Local Gravity," PoS CORFU 2014 (2015) 162 [arXiv:1508.07410 [hepth]].

[30] S. Talaganis and A. Mazumdar, "Towards UV Finiteness of Infinite Derivative Theories of Gravity and Field Theories," arXiv:1704.08674 [hep-th].

[31] J. Edholm, A. S. Koshelev and A. Mazumdar, "Behavior of the Newtonian potential for ghost-free gravity and singularity-free gravity," Phys. Rev. D 94 (2016) no.10, 104033 doi:10.1103/PhysRevD.94.104033 [arXiv:1604.01989 [gr-qc]].

[32] V. P. Frolov and A. Zelnikov, "Head-on collision of ultrarelativistic particles in ghost-free theories of gravity," Phys. Rev. D 93 (2016) no.6, 064048 doi:10.1103/PhysRevD.93.064048 [arXiv:1509.03336 [hep-th]].

[33] A. Mazumdar, S. Talaganis and A. Teimouri, "Hamiltonian Analysis for Infinite Derivative Field Theories and Gravity," arXiv:1701.01009 [hep-th]

[34] T. Biswas, T. Koivisto and A. Mazumdar, "Towards a resolution of the cosmological singularity in nonlocal higher derivative theories of gravity," JCAP 1011 (2010) 008 doi:10.1088/1475-7516/2010/11/008 [arXiv:1005.0590 [hep-th]].

[35] A. Conroy, A. S. Koshelev and A. Mazumdar, "Geodesic completeness and homogeneity condition for cosmic inflation," Phys. Rev. D 90 (2014) no.12, 123525 doi:10.1103/PhysRevD.90.123525 [arXiv:1408.6205 [gr$\mathrm{qc}]$.

[36] A. Conroy, A. S. Koshelev and A. Mazumdar, "Criteria for resolving the cosmological singularity in Infinite Derivative Gravity," arXiv:1605.02080 [gr-qc].

[37] A. Conroy, "Infinite Derivative Gravity: A Ghost and Singularity-free Theory," arXiv:1704.07211 [gr-qc].

[38] L. Perivolaropoulos, "Sub-millimeter Spatial Oscillations of Newton's Constant: Theoretical Models and Laboratory Tests," arXiv:1611.07293 [gr-qc].

[39] T. Biswas, A. Mazumdar and W. Siegel, "Bouncing universes in string-inspired gravity," JCAP 0603 (2006) 009 doi:10.1088/1475-7516/2006/03/009 [hep-th/0508194].

[40] S. Hawking and G. Ellis, "The Large Scale Structure of Space-Time" (1973) Cambridge University Press

[41] R. P. Woodard, "Ostrogradsky's theorem on Hamiltonian instability," Scholarpedia 10 (2015) no.8, 32243 doi:10.4249/scholarpedia.32243 [arXiv:1506.02210 [hepth]].

[42] A. Conroy, T. Koivisto, A. Mazumdar and A. Teimouri, "Generalized quadratic curvature, non-local infrared modifications of gravity and Newtonian potentials," Class. Quant. Grav. 32 (2015) no.1, 015024 doi:10.1088/0264-9381/32/1/015024 [arXiv:1406.4998 
[hep-th]].

[43] Schwartz MD. Quantum Field Theory and the Standard Model. 2013

[44] Claus Kiefer. Quantum gravity, Volume 155 of International series of mono- graphs on physics. Oxford Univ. Pr., Oxford, UK, 2012
[45] C. Gao, "Generalized modified gravity with the second order acceleration equation," Phys. Rev. D 86 (2012) 103512 doi:10.1103/PhysRevD.86.103512 [arXiv:1208.2790 [gr-qc]].

[46] S. Deser and R. P. Woodard, "Nonlocal Cosmology," Phys. Rev. Lett. 99 (2007) 111301 doi:10.1103/PhysRevLett.99.111301 [arXiv:0706.2151 [astro-ph]]. 